INPLASY

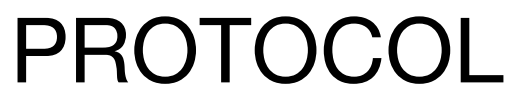

To cite: Cheng et al.

Effectiveness comparisons of various therapies for FIGO stage IB2/IIA2 cervical cancer: a Bayesian network

meta-analysis. Inplasy protocol 2020100069. doi:

10.37766/inplasy2020.10.0069

Received: 19 October 2020

Published: 19 October 2020

Corresponding author: Jing Cheng

chengjing62020@163.com

Author Affiliation:

The first Affiliated Hospital of Kunming Medical University

Support: No funds.

Review Stage at time of this submission: Formal screening of search results against eligibility criteria.

Conflicts of interest:

The authors declare no conflict of interests.

\section{Effectiveness comparisons of various therapies for FIGO stage IB2/IIA2 cervical cancer: a Bayesian network meta-analysis}

Cheng, J1; Liu, BB2; Xu, L3; Wang, B4; Long, XC5; Li, ZH6; Wu, RT7.

Review question / Objective: At present, domestic and foreign studies have shown that there is still controversy over the treatment of patients with cervical cancer stage IB2/IIA2. In this paper, the bayesian reticular meta-analysis method was adopted to find out the best treatment scheme most suitable for such patients. This research may provide an excellent resource for future cervical cancer efforts, or it may serve as a springboard for unknown creative ventures. Condition being studied: Cervical cancer stage IB2/IIA2.

Information sources: PubMed, Embase, the Cochrane Central Register of Controlled Trials, were used to perform an exhaustive search.

INPLASY registration number: This protocol was registered with the International Platform of Registered Systematic Review and Meta-Analysis Protocols (INPLASY) on 19 October 2020 and was last updated on 19 October 2020 (registration number INPLASY2020100069).

\section{INTRODUCTION}

Review question / Objective: At present, domestic and foreign studies have shown that there is still controversy over the treatment of patients with cervical cancer stage IB2/IIA2. In this paper, the bayesian reticular meta-analysis method was adopted to find out the best treatment scheme most suitable for such patients. This research may provide an excellent resource for future cervical cancer efforts, or it may serve as a springboard for unknown creative ventures. 
Condition being studied: Cervical cancer stage IB2/IIA2.

\section{METHODS}

Participant or population: 1) Premenopausal or Postmenopausal Women (aged from 18 to 75 years ); 2) Women had a diagnosis in stage IB2 or IIA2 Cervical Carcinoma based on FIGO.

Intervention: 1) Concomitant chemoradiotherapy (CCRT); 2) Radical surgery(RS); 3) Adjuvant hysterectomy following chemoradiotherapy (AH+CCRT); 4) Neoadjuvant chemotherapy followed by radical surgery(NACT+RS); 5) Adjuvant radiotherapy following Radical surgery (AR+RS).

Comparator: 1) Other treatments for cervical cancer; 2) named control group.

Study designs to be included: Randomized controlled trials has been published without year and language restriction.

Eligibility criteria: Inclusion criteria are implemented according to PICOS.

Information sources: PubMed, Embase, the Cochrane Central Register of Controlled Trials, were used to perform an exhaustive search.

Main outcome(s): 1) The survival rate; 2) The recurrence rate; 3 ) The mortality rate; 4) The complications.

Quality assessment / Risk of bias analysis: We appraised and classified the risk of bias (ROB) of each included study according to the Cochrane Risk of Bias tool and classified them, as per criteria defined a priority. Seven domains regarding ROB were assessed independently by two authors were judging each study into a high, low or unclear risk of bias, including random sequence generation, allocation concealment, blinding of participants and personnel, blinding of outcome assessment, incomplete outcome data, selective reporting, and other bias.
Strategy of data synthesis: The quality of evidence was assessed using the GRADE approach. Factors such as high likelihood of methodological bias, unexplained heterogeneity, inconsistency or imprecision of results were deemed to decrease the quality level of a body of evidence. The following gratings were used: high, moderate, low, very low.

Subgroup analysis: If there is heterogeneity between studies, we will conduct a subgroup analysis.

Sensibility analysis: If there is heterogeneity between studies, we will conduct a sensibility analysis.

Country(ies) involved: China.

Keywords: Radiotherapy, chemotherapy, radical surgery, Cervical cancer IB2/IIA2, Bayesian Network Meta-analysis

Dissemination plans: The results of this network meta-analysis will be published in a peer-reviewed journal.

Contributions of each author:

Author 1 - Jing Cheng.

Author 2 - BeiBei Liu.

Author 3 - Lin Xu.

Author 4 - Biao Wang. 